\title{
Topical Drug Delivery to the Posterior Segment of the Eye: Addressing the Challenge of Preclinical to Clinical Translation
}

\author{
Gerard A. Rodrigues ' David Lutz' ${ }^{1}$ Jie Shen ${ }^{2} \cdot$ Xiaoda Yuan ${ }^{3} \cdot$ Hong Shen $^{3}$ • \\ James Cunningham ${ }^{3} \cdot$ Hongwen M. Rivers ${ }^{3,4}$
}

Received: 17 August 2018 / Accepted: 3 October 2018 / Published online: 29 October 2018

(C) The Author(s) 2018

\begin{abstract}
Topical delivery of therapeutics to the posterior segment of the eye remains the "holy grail" of ocular drug delivery. As an example, anti-vascular endothelial growth factor biologics, such as ranibizumab, aflibercept, and bevacizumab, are delivered by intravitreal injection to treat neovascular age-related macular degeneration and, although these drugs have revolutionized treatment of the disease, less invasive alternatives to intravitreal injection are desired. Multiple reports in the literature have demonstrated topical delivery of both small and large molecules to the back of the eye in small animal models. Despite this progress, successful translation to larger species, and ultimately humans, has yet to be demonstrated. Selection of animal models with relevant ocular anatomy and physiology, along with appropriate experimental design, is critical to enable more relevant feasibility assessments and increased probability of successful translation.
\end{abstract}

KEY WORDS formulation · in vivo evaluation · posterior segment · topical delivery translation

\section{ABBREVIATIONS}

AMD Age-related macular degeneration

CPP Cell-penetrating peptide

Guest Editors: Hovhannes J Gukasyan, Shumet Hailu, and Thomas Karami

Hongwen M. Rivers

rivers_hongwen@allergan.com

Biological Research, Allergan plc, Invine, California 92612, USA

2 Nonclinical and Translational Sciences, Allergan plc, Invine, California 92612, USA

3 Pharmaceutical Development, Allergan plc, Irvine, California 926 12, USA

4 Allergan plc, 2525 Dupont Drive, Invine, California 926।2-I531, USA

$\begin{array}{ll}\text { CNV } & \text { Choroidal neovascularization } \\ \text { TAT } & \begin{array}{l}\text { Human immunodeficiency virus (HIV) } \\ \text { transactivator of transcription }\end{array} \\ \text { POD } & \text { Peptide for ocular delivery } \\ \text { VEGF } & \text { Vascular endothelial growth factor } \\ \text { VEGFR-2 } & \text { VEGF receptor-2 }\end{array}$

\section{INTRODUCTION}

Topical instillation of eye drops is non-invasive and the most common route for administering therapeutics to the eye. Although this route is a viable method of drug delivery for the treatment of anterior segment diseases, it remains a major challenge to efficiently deliver drugs topically to treat posterior segment diseases such as age-related macular degeneration (AMD) and diabetic macular edema. Static and dynamic barriers limit penetration of therapeutic molecules into the ocular tissues $(1,2)$. Static barriers to drug transport include the corneal epithelium, conjunctival epithelium, sclera, choroid, Bruch's membrane, and retinal pigmented epithelium; these work together with dynamic barriers such as choroidal and conjunctival blood flow, lacrimation, and lymphatic drainage and efflux to efficiently reject foreign substances and pathogens. As a result, bioavailability is low following topical dosing of eye drops, with typically less than $3 \%$ of topically administered drug reaching the aqueous humor (1) and even less reaching the posterior segment, resulting in subtherapeutic drug concentrations in these tissues (3).

Despite the large unmet need and market opportunity, topical delivery of hydrophilic macromolecule drugs such as therapeutic proteins to the posterior segment remains particularly challenging. The current standard of care for the treatment of AMD is intravitreally administered anti-vascular endothelial growth factor (anti-VEGF) biologics. A number of registration trials have established the recommended frequency of these injections, which is typically monthly or bimonthly 
depending on the drug. In addition to the significant treatment burden for patients and caregivers, frequent intravitreal injections increase the risk of complications including endophthalmitis, cataracts, retinal detachment, and vitreous hemorrhage. In real-world experience, patients may receive fewer treatments than those participating in clinical trials and, as a consequence, have poorer-than-expected treatment outcomes (4). Clearly, achieving effective delivery with a less invasive route of administration could provide significant benefit to patients. Representative studies that have investigated topical administration of molecules in preclinical models are listed in Table I. This article discusses the key considerations in evaluating topical drug delivery for treating retinal diseases, with an emphasis on translation from preclinical models to humans.

\section{Preclinical Evaluation: Animal Models and Translatability}

Many preclinical studies claiming successful topical delivery of small molecules or proteins to the retina have used rodent models (Table I). Advantages include the availability of established and well accepted pharmacological models, and practical considerations such as cost and availability. Despite these advantages, the rodent eye is anatomically dissimilar to the human eye, and resulting differences in the distribution and elimination of drugs can have a significant impact on the pharmacodynamic response. Thus, efficacy data generated using rodent models should be interpreted with caution.
The importance of choosing the appropriate preclinical animal model is underscored by multiple failures of topically administered compounds in clinical trials, despite promising preclinical data in rodents. For example, TG100801, a smallmolecule multikinase inhibitor, was shown to suppress laserinduced choroidal neovascularization (CNV) in a mouse mod$\mathrm{el}$, as well as edema in a rat model of retinal vein occlusion, following topical administration (5). TG100801 is a prodrug selected for its ability to penetrate the cornea; the active inhibitor is then generated by hydrolysis (5). However, no pharmacodynamic studies were conducted in a larger species, and when tested in humans, although well tolerated, TG100801 failed to demonstrate efficacy in AMD patients (ClinicalTrials. gov identifier: NCT00509548). Similarly, pazopanib, a smallmolecule multikinase inhibitor formulated as an eye drop at $5 \mathrm{mg} / \mathrm{ml}$ and dosed topically twice daily, significantly decreased leakage in a laser-induced CNV model in rats by $\sim 90 \%$ (6). However, when it was later tested as a $10-\mathrm{mg} / \mathrm{ml}$ eye drop dosed four times daily for 12 weeks in patients with subfoveal GNV secondary to AMD, it did not decrease central retinal thickness (7). A very similar outcome was recently reported for acrizanib, a VEGF receptor-2 (VEGFR-2) inhibitor specifically designed for topical ocular delivery to treat neovascular AMD (8). The rodent CNV model (both mouse and rat) was used as the primary means of developing structure-activity relationships and screening VEGFR-2 inhibitors with potent in vitro activities for topical delivery. Acrizanib showed a 99\% inhibitory effect following three times daily dosing as a $1 \%$ suspension in the mouse GNV

Table I Examples of Topically Administered Molecules Investigated in Preclinical Models

\begin{tabular}{|c|c|c|c|}
\hline Compound & Formulation & Preclinical data & Reference \\
\hline TGI0080। & Solution & CNV model in mouse, edema in rat & (5) \\
\hline Pazopanib & Solution & CNV model in rat & $(67)$ \\
\hline Acrizanib & Suspension & CNV model rat and mouse & (8) \\
\hline Memantine & Solution & Drug levels in retina of rabbit & $(1)$ \\
\hline Dorzoamide & Solution & $\begin{array}{l}\text { Drug levels and carbonic anhydrase activity in corneal } \\
\text { endothelial cells, ciliary body, lens epithelial cells, } \\
\text { and retina in rabbit }\end{array}$ & $(13)$ \\
\hline Dexamethasone & lontophoresis & Drug levels in retina and vitreous of rabbit & $(14)$ \\
\hline Bevacizumab & Solution & $\begin{array}{l}\text { Drug levels in iris/ciliary body, vitreous, retina/choroid, } \\
\text { and plasma in rabbit }\end{array}$ & $(16)$ \\
\hline Anti-intercellular adhesion molecule-I antibody & Solution by osmotic pump & $\begin{array}{l}\text { Drug levels and VEGF-induced leukostasis in the choroid } \\
\text { and retina in rabbit }\end{array}$ & $(17)$ \\
\hline 28-kD single-chain antibody fragment & Sodium caprate & Drug levels in vitreous in rabbit & $(18)$ \\
\hline Bevacizumab & Annexin A5-based liposomes & Drug levels in retina of rat and rabbit & $(19)$ \\
\hline Transforming growth factor beta I & Annexin A5-based liposomes & Drug levels in vitreous in rabbit & (20) \\
\hline Acidic fibroblast growth factor & CPP (TAT) & Ischemia reperfusion model in rat & $(22)$ \\
\hline Calpain inhibitory peptide & CPP (TAT) & Drug levels in retina of rabbit & $(23)$ \\
\hline Green fluorescent protein & CPP (POD) & Drug levels in cornea of mouse & $(24)$ \\
\hline Bevacizumab & CPP (R6) & $\begin{array}{l}\text { Drug levels in vitreous and retina in rat and CNV model } \\
\text { in mouse }\end{array}$ & $(25)$ \\
\hline
\end{tabular}


model, whereas 94\% inhibition was achieved when dosed as a $3 \%$ suspension twice a day in the rat CNV model. Despite the positive data in both rodent models, acrizanib did not demonstrate efficacy in a proof of concept study in patients with neovascular AMD (ClinicalTrials.gov identifier: NCT02355028). In spite of these failures, topical delivery of multikinase inhibitors to the posterior segment continues to be an active area of research. For example, a topical suspension formulation of a multikinase inhibitor, PAN-90806, is currently being tested in AMD patients in a phase I/II trial (ClinicalTrials.gov identifier: NGT03479372).

Compared with rodents, the relevant anatomical and physiological parameters in rabbits are more similar to those in humans (9). Rabbits share ocular features with humans including a comparable size, vitreal volume, and internal structure, and thus a similar diffusional path for topically administered compounds to reach the posterior segment (9). Additionally, the intravitreal pharmacokinetic parameters in rabbits have shown predictable correlations with those in humans (10). The rabbit is also relatively easy to handle and is the most economical of the larger species models. Importantly, an increasing number of rabbit models of ocular diseases, including AMD, have been established (9). Even preclinical data generated through use of a larger animal species such as rabbits should be interpreted with caution due to anatomical/physiological differences that have the potential to impact drug disposition. For example, rabbits have a lower blinking rate than humans, which would be expected to increase the residence time of topically administered drug formulations and potentially affect the bioavailability of drugs in intraocular tissues (11). In addition, the proportionally larger anterior segment and more viscous vitreous humor in rabbit relative to human eyes may also affect distribution of drugs that rely more predominantly on a corneal route of diffusion (Fig. 1). Finally, animals with healthy eyes are typically used in pharmacokinetic studies. This may result in underestimation of drug clearance when extrapolated to diseased human eyes with a compromised blood-retinal barrier (12).

In addition to selection of an appropriate animal model, proper study design is critical to avoid incorrect interpretation of study results with regard to drug distribution. Topically administered drugs may enter the eye via the cornea and/or the conjunctival epithelium. Penetration of drugs to posterior segment tissues following corneal absorption involves transit through the anterior segment before reaching the vitreous and the retina. Following absorption into the conjunctiva, drugs can either diffuse through the sclera or the cornea, or alternatively, be cleared into the systemic circulation before reaching the vitreous and retina (1) (Fig. 1). In our experience, assessment of drug levels and/or efficacy in the contralateral, untreated eye is critical to eliminate a potential confounding factor of drug distribution from the systemic circulation following topical administration. Given the much larger blood volume in humans vs. rabbits $(\sim 51$ in an adult human $v s . \sim 0.12 \mathrm{l}$ in a 2-kg adult rabbit), drug distribution to the back of the eye via systemic circulation following topical administration in rabbits may result in tissue levels that do not translate to humans.

To date, the reported preclinical successes of posterior segment drug exposure from topical dosing in a rabbit eye have been primarily observed with small molecules (3) (Table I). For example, topical instillation of $35 \mu \mathrm{l}$ of $0.1 \%$ memantine aqueous solution twice daily for 7 days was able to achieve drug levels in the retinal tissue sufficient for retinal neuroprotection in a rabbit model (1). In another example, retinal concentrations of dorzolamide adequate for inhibition of carbonic anhydrase isoenzyme II in rabbits were achieved using a oncedaily topical dose of $1 \%$ aqueous dorzolamide $\mathrm{HCl}$ solution (13). Iontophoresis, the use of electrical current to enhance tissue penetration, is a topical drug delivery approach that has shown some promise. Although the number of studies using transcorneal iontophoresis for posterior segment delivery is limited, there are multiple examples of successful transscleral iontophoresis of small molecules, including antibiotics and steroids, into the vitreous of rabbits (14). Iontophoretic dexamethasone was advanced into a phase $\mathrm{Ib} / \mathrm{II}$ a clinical trial (ClinicalTrials.gov identifier: NCT02485249) for patients with macular edema before the trial was terminated due to enrollment issues.

While there has been some success in the topical delivery of small molecules to the posterior segment in a large eye, the hurdles for biologics are higher given the much larger molecular weight and polarity. It has been shown that scleral permeability decreases with increasing molecular weight and molecular radius (15). On the other hand, biologics typically have higher affinities for their target than small molecules. Thus, the challenges associated with lower corneal or scleral penetration may be offset to a certain degree by a lower drug concentration needed at the target to achieve efficacy. However, even the potent biologic bevacizumab failed to achieve therapeutic concentrations in the retina and vitreous of rabbits following aggressive topical dosing of $1.25 \mathrm{mg} /$ $0.05 \mathrm{ml}$ six times daily for a week (16). Ambati et al. (17) reported successful transscleral delivery of a monoclonal antibody directed against intercellular adhesion molecule 1 to the retina and choroid in rabbits without detectable systemic exposure, resulting in inhibition of VEGF-induced leukostasis. However, this required use of an osmotic pump delivering drug constantly over a $24-\mathrm{h}$ period to the superotemporal scleral surface (17).

To address the challenge of topical protein delivery, different formulation approaches have been explored to attempt to overcome barriers and reach therapeutically meaningful drug levels in the posterior segment (Table I). Williams et al. (18) reported detection of a $28-\mathrm{kDa}$ single-chain variable domain 
Fig. I Drug distribution pathways through the corneal and conjunctival/scleral routes following topical administration. Reprinted from Advanced Drug Delivery Review, Vol 57, Hughes PM, Olejnik $O$, Chang-Lin JE, Wilson CG, Topical and systemic drug delivery to the posterior segments, Pages 20 I0-32, Copyright 2005, with permission from Elsevier.

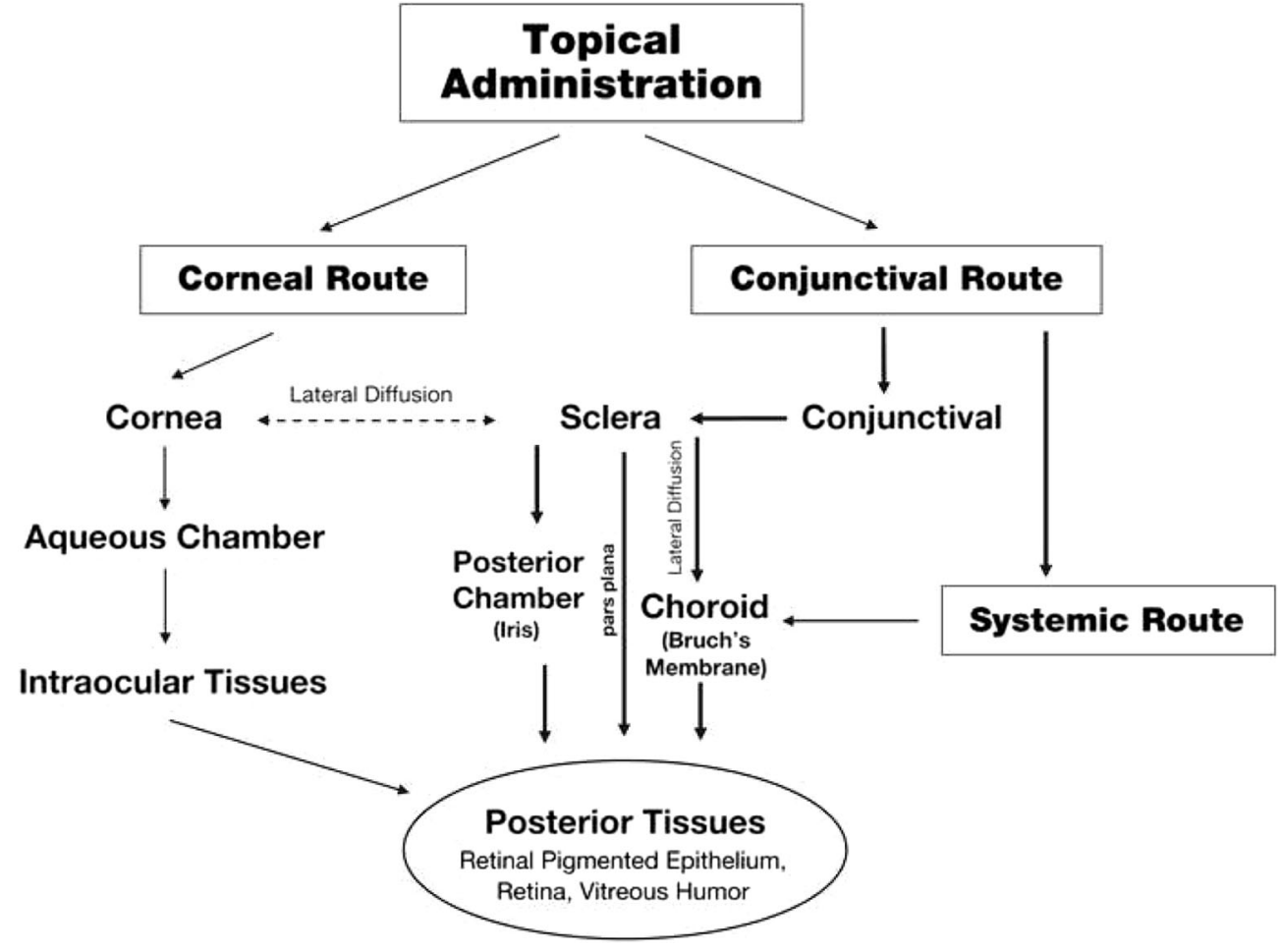

antibody fragment in the rabbit vitreous at concentrations of 50-150 ng/ml $12 \mathrm{~h}$ after topical dosing of the antibody fragment with a permeability enhancer, sodium caprate. Interestingly, penetration of its corresponding full-length (IgG) antibody into the vitreous was not observed (18). Davis et al. (19) reported delivery of physiologically relevant concentrations of bevacizumab to the back of the eye in both rats and rabbits using annexin A5-associated liposomes. Similarly, Platania et al. (20) reported the use of an annexin A5-based liposome formulation to deliver high levels of transforming growth factor beta 1 to the rabbit vitreous following topical administration. The use of cell-penetrating peptides (CPPs) is another formulation approach that has been investigated for ocular delivery of proteins and peptides (21). However, preclinical studies to date using this approach have been largely limited to rodent models. For example, Wang et al. (22) used the CPP HIV transactivator of transcription (TAT) to deliver acidic fibroblast growth factor to the rat retina following topical administration at levels sufficient to protect the retina from ischemia-reperfusion injury. Similarly, Ozaki et al. (23) demonstrated delivery of topically administered calpain inhibitory peptide conjugated to TAT to the posterior segment of the rat eye. Another relevant example is the use of a CPP, peptide for ocular delivery (POD), conjugated to green fluorescent protein to enable its uptake by corneal epithelium when the formulation was topically administered to mouse eyes (24). Most recently, de Cogan et al. (25) reported that therapeutic levels of bevacizumab in the posterior segment were achieved in rats following topical administration when the antibody was combined with a CPP, poly-arginine-6. Notwithstanding these encouraging results in rodents, the translational application of these platforms is still in its infancy. Moreover, the enhancement of corneal permeability through the use of agents such as sodium caprate has the potential to cause ocular toxicity associated with compromised interepithelial tight junctions (3). Therefore, in addition to the evaluation of efficacy, thorough safety assessment in a non-rodent species is warranted for these novel formulationbased methods of topical delivery (26).

\section{CONCLUSIONS}

Although topical ocular drug administration represents a less invasive and therefore safer route of dosing than intraocular injections, clinical success in delivering drugs to the posterior segment by this route remains elusive. There are now multiple examples of preclinical pharmacokinetic and efficacy data generated in rodent models failing to translate to humans. The absence of translation from rodents to humans is not surprising when anatomical and fluid dynamic differences in the eye across species are considered. Based on parameters including, but not limited to, corneal thickness, aqueous humor volume and flow rate, vitreal volume, and circumferential or linear distance from the ocular surface to the back of the eye, one would expect delivery of drugs to the posterior segment via topical dosing to be more difficult in humans than in rodents. Unfortunately, rodents remain the species of choice 
for many feasibility studies evaluating topical delivery. We advocate for the use of larger species such as rabbits, dogs, pigs, or monkeys when testing novel drug delivery approaches to maximize the probability of predictive preclinical assessment and successful clinical translation. In addition, assessment of ocular pharmacokinetics in both the treated and contralateral eyes as well as in plasma is important to inform the delivery mechanism. Lastly, thorough safety assessment, which can and should be considered as part of the study design, is also critical. With the continued innovation of novel delivery approaches, we remain hopeful that topical delivery of drugs, including biologics, to the posterior segment of the eye may become a reality in the future to address a significant unmet medical need.

Open Access This article is distributed under the terms of the Creative Commons Attribution 4.0 International License (http://creativecommons.org/licenses/by/4.0/), which permits unrestricted use, distribution, and reproduction in any medium, provided you give appropriate credit to the original author(s) and the source, provide a link to the Creative Commons license, and indicate if changes were made.

\section{REFERENCES}

1. Hughes PM, Olejnik O, Chang-Lin JE, Wilson CG. Topical and systemic drug delivery to the posterior segments. Adv Drug Deliv Rev. 2005;57(14):2010-32.

2. Barar J, Javadzadeh AR, Omidi Y. Ocular novel drug delivery: impacts of membranes and barriers. Expert Opin Drug Deliv. 2008;5(5):567-81.

3. Boddu SH, Gupta H, Patel S. Drug delivery to the back of the eye following topical administration: an update on research and patenting activity. Recent Pat Drug Deliv Formul. 2014;8(1):27-36.

4. Holz FG, Tadayoni R, Beatty S, Berger A, Cereda MG, Cortez R, et al. Multi-country real-life experience of anti-vascular endothelial growth factor therapy for wet age-related macular degeneration. $\mathrm{Br}$ J Ophthalmol. 2015;99(2):220-6.

5. Doukas J, Mahesh S, Umeda N, Kachi S, Akiyama H, Yokoi K, et al. Topical administration of a multi-targeted kinase inhibitor suppresses choroidal neovascularization and retinal edema. J Cell Physiol. 2008;216(1):29-37.

6. Yafai Y, Yang XM, Niemeyer M, Nishiwaki A, Lange J, Wiedemann $\mathrm{P}$, et al. Anti-angiogenic effects of the receptor tyrosine kinase inhibitor, pazopanib, on choroidal neovascularization in rats. Eur J Pharmacol. 201 1;666(1-3):12-8.

7. Singh R, Wurzelmann JI, Ye L, Henderson L, Hossain M, Trivedi $\mathrm{T}$, et al. Clinical evaluation of pazopanib eye drops in healthy subjects and in subjects with neovascular age-related macular degeneration. Retina. 2014;34(9):1787-95.

8. Adams CM, Anderson K, Artman G 3rd, Bizec JC, Cepeda R, Elliott J, et al. The discovery of $\mathrm{N}$-(1-methyl-5-(trifluoromethyl)1H-pyrazol-3-yl)-5-((6- ((methylamino)methyl)pyrimidin-4-yl)oxy)1H-indole-1-carboxamide (acrizanib), a VEGFR-2 inhibitor specifically designed for topical ocular delivery, as a therapy for neovascular age-related macular degeneration. J Med Chem. 2018;61(4):1622-35.

9. Zernii EY, Baksheeva VE, Iomdina EN, Averina OA, Permyakov SE, Philippov PP, et al. Rabbit models of ocular diseases: new relevance for classical approaches. CNS Neurol Disord Drug Targets. 2016;15(3):267-91.

10. Del Amo EM, Urtti A. Rabbit as an animal model for intravitreal pharmacokinetics: clinical predictability and quality of the published data. Exp Eye Res. 2015;137:111-24.

11. Owen GR, Brooks AC, James O, Robertson SM. A novel in vivo rabbit model that mimics human dosing to determine the distribution of antibiotics in ocular tissues. J Ocul Pharmacol Ther. 2007;23(4):335-42.

12. Shen J, Durairaj C, Lin T, Liu Y, Burke J. Ocular pharmacokinetics of intravitreally administered brimonidine and dexamethasone in animal models with and without blood-retinal barrier breakdown. Invest Ophthalmol Vis Sci. 2014;55(2):1056-66.

13. Inoue J, Oka M, Aoyama Y, Kobayashi S, Ueno S, Hada N, et al. Effects of dorzolamide hydrochloride on ocular tissues. J Ocul Pharmacol Ther. 2004;20(1):1-13.

14. Ambati J, Adamis AP. Transscleral drug delivery to the retina and choroid. Prog Retin Eye Res. 2002;21(2):145-51.

15. Ambati J, Canakis CS, Miller JW, Gragoudas ES, Edwards A, Weissgold DJ, et al. Diffusion of high molecular weight compounds through sclera. Invest Ophthalmol Vis Sci. 2000;41(5):1 181-5.

16. Nomoto H, Shiraga F, Kuno N, Kimura E, Fujii S, Shinomiya K, et al. Pharmacokinetics of bevacizumab after topical, subconjunctival, and intravitreal administration in rabbits. Invest Ophthalmol Vis Sci. 2009;50(10):4807-13.

17. AmbatiJ, Gragoudas ES, Miller JW, You TT, Miyamoto K, Delori FC, et al. Transscleral delivery of bioactive protein to the choroid and retina. Invest Ophthalmol Vis Sci. 2000;41(5):1 186-91.

18. Williams KA, Brereton HM, Farrall A, Standfield SD, Taylor SD, Kirk LA, et al. Topically applied antibody fragments penetrate into the back of the rabbit eye. Eye (Lond). 2005;19(8):910-3.

19. Davis BM, Normando EM, Guo L, Turner LA, Nizari S, O'Shea $\mathrm{P}$, et al. Topical delivery of Avastin to the posterior segment of the eye in vivo using annexin A5-associated liposomes. Small. 2014;10(8):1575-84.

20. Platania CBM, Fisichella V, Fidilio A, Geraci F, Lazzara F, Leggio GM, et al. Topical ocular delivery of TGF- $\beta 1$ to the back of the eye: implications in age-related neurodegenerative diseases. Int $\mathrm{J}$ Mol Sci. 2017;18(10):2076.

21. Fonseca SB, Pereira MP, Kelley SO. Recent advances in the use of cell-penetrating peptides for medical and biological applications. Adv Drug Deliv Rev. 2009;61(11):953-64.

22. Wang Y, Lin H, Lin S, Qu J, Xiao J, Huang Y, et al. Cellpenetrating peptide TAT-mediated delivery of acidic FGF to retina and protection against ischemia-reperfusion injury in rats. J Cell Mol Med. 2010;14(7):1998-2005.

23. Ozaki T, Nakazawa M, Yamashita T, Ishiguro S. Delivery of topically applied calpain inhibitory peptide to the posterior segment of the rat eye. PLoS One. 2015;10(6):e0130986.

24. Johnson LN, Cashman SM, Read SP, Kumar-Singh R. Cell penetrating peptide POD mediates delivery of recombinant proteins to retina, cornea and skin. Vis Res. 2010;50(7):686-97.

25. de Cogan F, Hill LJ, Lynch A, Morgan-Warren PJ, Lechner J, Berwick MR, et al. Topical delivery of anti-VEGF drugs to the ocular posterior segment using cell-penetrating peptides. Invest Ophthalmol Vis Sci. 2017;58(5):2578-90.

26. Short BG. Safety evaluation of ocular drug delivery formulations: techniques and practical considerations. Toxicol Pathol. 2008;36(1):49-62. 